\title{
Spin-down of relativistic stars with phase transitions and PSR J0537-6910
}

\author{
N. K. Spyrou and N. Stergioulas
}

\author{
Astronomy Department, Aristoteleion University of Thessaloniki, 541.24 Thessaloniki, Macedonia, Greece \\ e-mail: spyrou@astro.auth.gr, niksterg@astro.auth.gr
}

Received 22 April 2002 / Accepted 26 August 2002

\begin{abstract}
Using a highly accurate numerical code, we study the spin down of rotating relativistic stars undergoing a quark deconfinement phase transition. Such phase transitions have been suggested to yield an observable signal in the braking index of spinning-down pulsars which is based on a "backbending" behaviour of the moment of inertia. We focus on a particular equation of state that has been used before to study this behaviour, and find that for the population of normal pulsars the moment of inertia does not exhibit a backbending behaviour. In contrast, for supramassive millisecond pulsars a very strong backbending behaviour is found. Essentially, once a quark core appears in a spinning-down supramassive millisecond pulsar, the star spins up and continues to do so until it reaches the instability to collapse. This strong spin-up behaviour makes it easier to distinguish a phase transition in such pulsars: a negative first time-derivative of the rotational period, $\dot{P}<0$, suffices and one does not have to measure the braking index. In the spin-up era, the usually adopted spin-down power law fails to describe the evolution of the angular velocity. We adopt a general-relativistic spin-down power law and derive the equations that describe the angular velocity and braking index evolution in rapidly rotating pulsars. We apply our numerical results to the fast young pulsar J0537-6910 in SNR N157B, which has been suggested to have (if spun down by magnetic dipole radiation only) an extremely small initial spin period. The inclusion of a quark-hadron phase transition can yield a significantly larger initial spin period of $6 \mathrm{~ms}$ (in our example), which is in better agreement with theoretical expectations. Finally, we suggest that the frequent rate of glitches in PSR J0537-6910 could be related to the fact that it is the fastest Crab-like pulsar, so that a pure quark core may have formed recently in its lifetime.
\end{abstract}

Key words. stars: pulsars: general - stars: rotation

\section{Introduction}

The properties of the highest-density region of matter in the interior of relativistic stars have been studied to date by several different theoretical models, which yield very different macroscopic properties of compact stars. Many new interactions have been proposed to occur at the highest densities attained, ranging from the occurrence of hyperons, to quark deconfinement, to the formation of kaon condensates and H-matter (see Weber 2001; Heiselberg 2002, for recent reviews). Observational constraints on pulsar properties are currently still too weak to allow to distinguish which (if any) of all the proposed theoretical descriptions of the interior of relativistic stars is the correct one. A solution to the problem of determining the correct equation of state in the central region of a compact star must come from new, more accurate observations of their properties and any new observational method that will help in this direction is more than welcome.

One new observational method, that could be interesting in the case that the equation of state (EOS) features a deconfinement phase transition to quark matter, has been

Send offprint requests to: N. Stergioulas, e-mail: niksterg@astro.auth.gr proposed by Glendenning et al. (1997), (hereafter GPW). Specifically, when a rapidly rotating pulsar spins down, its central density increases with time. At a certain central density, a mixed quark-hadron phase can appear (or can already be present in the pulsar). For some chosen parameters of the equation of state and of the baryonic mass of the pulsar, a pure quark core appears when the pulsar reaches very high densities. In GPW a particular spin-down sequence was studied, that in the nonrotating limit reached nearly at the maximum mass allowed by the chosen EOS. For this sequence, it was noticed that, when the pure quark core appears, the star undergoes an brief era of spin-up (brief, compared to the pulsar's lifetime) and its braking index shows an anomalous behaviour (it becomes singular at a certain rotation rate). This change in the braking index has been proposed in GPW to be potentially observable. The anomalous behaviour of the spin evolution is traced back to a "backbending" of the moment of inertia (as a function of angular velocity).

Other authors (Heiselberg \& Hjorth-Jensen 1998; Chubarian et al. 2000) have further investigated the observational consequences in the models proposed in GPW and in Glendenning \& Weber (2000). In all these studies the 
relativistic slow-rotation approximation is used ${ }^{1}$. In the present work, we re-investigate the deconfinement phase-transition of spinning-down pulsars, using fully relativistic, rapidly rotating models and find that, when the quark-core appears (in a sequence of models similar to the one used in GPW), the backbending in the moment of inertia is generically absent for normal pulsars, but very strong for supramassive millisecond pulsars. Furthermore, our numerical results suggest that in the limiting region between normal and suprammasive pulsars, the backbending behaviour is sensitive to truncation errors of the applied numerical scheme. The moment of inertia is a very sensitive function of angular velocity and must be computed with high accuracy. Truncation errors, such as a limited number of points in the EOS table, or the use of the slow-rotation approximation can be large enough to systematically affect the computation of the moment of inertia.

The case of supramassive millisecond pulsars is very interesting, as the spin-up era lasts from the onset of the quarkcore appearence to their collapse to black holes, i.e. for their remaining lifetime. For supramassive millisecond pulsars created via accretion-induced spin-up, the spin-up era could last for the most part of their lifetime, if a quark-hadron phase transition occurs. Thus, the first time-derivative of the rotational period can be used as a tool for observing quark-hadron phase transitions and one does not have to rely on the measurement of the braking index. We emphasize that the extended spin-up era in supramassive millisecond pulsars is not restricted for the case of a quark-hadron phase transition, but it can also occur for other types of phase transitions, such as a phase transition to a pion condensate. An example is EOS M (Pandharipande $\&$ Smith 1975) for which an extended spin-up with angular momentum loss (for supramassive, constant baryonic mass sequences) has been found in Cook et al. (1994). On the other hand, the absence of spin-up in the currently known millisecond pulsar population favours the absence of large phase transitions or implies that the EOS is such that millisecond pulsars created via accretion-induced spin-up are probably not supramassive.

In previous studies of spinning-down pulsars that were limited to the slow-rotation approximation (including up to $O\left(\Omega^{2}\right)$ rotational effects) expressions for the evolution of angular velocity and braking index were derived (see Glendenning 1997). However, we find that these expressions are incomplete, as changes in the gravitational mass of the star (other than changes in the kinetic energy) were not taken into account in the assumed spin-down law. Here, we adopt a general-relativistic version of the spin-down law, which dictates that the energy lost in the form of e.g. magnetic dipole radiation or gravitational waves is not lost to the expense of the kinetic energy only, but to the expense of the total mass-energy of the star. Hence, making use of a relation due to Bardeen (1970), which expresses the first law of thermodynamics along sequences of constant

\footnotetext{
${ }^{1}$ After this work was completed, we learned of a new study using rapidly rotating relativistic stars, with a numerical code related to ours, (Cheng et al. 2002), in which the backbending behaviour of the moment of inertia is shown to be sensitive to the assumed properties of the crust.
}

baryonic mass, we derive expressions for the angular velocity and braking index evolution, which are valid for any rotation rate. In addition, our adopted spin-down law is also valid during a spin-up era, while it's Newtonian, slow-rotation limiting expression (which is usually adopted in the literature) is not valid during spin-up.

As an application of our numerical investigations, we compute a possible value for the initial period of pulsar J05376910 , assuming that the high-density EOS exhibits a quarkdeconfinement phase transition and that the star spins down only due to magnetic dipole radiation. Without the assumption of a phase transition, previous studies suggested that the initial spin of the pulsar would be extremely small (for a braking index of $n=3$ ) (Marshall et al. 1998). Here we show that the presence of the phase transition can yield a more reasonable initial period for this pulsar, without having to assume a braking index smaller than $n=3$. In our example, we obtain a significantly larger initial period of $6 \mathrm{~ms}$, which is in better agreement with theoretical expectations. Furthermore, this pulsar has displayed a large number of giant glitches (Gotthelf et al. 2001). We propose that these could be due to the appearance of a pure quark core in the center of the star, which results in an increased rate of contraction of the star during its spindown. In contrast, rapidly rotating pulsars do not exhibit such giant glitches, when their central density is less than the critical density for the appearance of the quark core. The fact that pulsar J0537-6910 displays such glitches more frequently than other Crab-like ${ }^{2}$ pulsars may be related to the fact that it is the most rapidly spinning of all Crab-like pulsars and thus a pure quark core could have appeared more recently in its lifetime.

The outline of the paper is as follows: In Sect. 2, we describe the chosen equation of state and its numerical refinement, while in Sect. 3 we obtain the equilibrium properties of a specific sequence of spinning-down compact stars. The behaviour of the moment of inertia and gravitational mass along the sequence is described in Sect. 4 and thermodynamic consistency is used as an independent test in Sect. 5. In Sect. 6 expressions for the evolution of the angular velocity and the braking index are derived, and in Sect. 7 the initial period of PSR J0537-6910 is estimated. Finally, in Sect. 8, we comment on the expected event rate of the considered signal. We conclude with a discussion of our results in Sect. 9.

\section{Equation of state and numerical refinement}

The EOS used in GPW features a mixed quark-hadron phase, in between pure hadron and pure quark phases. The mixed phase is obtained by applying the Gibbs criteria for two-component systems, abandoning local (but not global) charge-neutrality (Glendenning 1992). This yields a second-order phase transition between each region. However, it has been suggested (see Heiselberg \& Hjorth-Jensen 1999) that the mixed phase can exist only if the interface tension between quarks and hadrons is not too large. But, on the other hand, the interface tension may not be a free parameter, but it could have the freedom to

\footnotetext{
${ }^{2}$ In this paper, "Crab-like" only refers to the young age of a pulsar and to its observational association with a supernova remnant.
} 
adjust (via a minimization prinicple) to the nature of the two phases (Glendenning 2002), see Christiansen et al. (2000) for a relevant example.

The specific EOS we use is the quark deconfinement EOS given in Table 9.2 of Glendenning (1997) (same as in GPW) in which, however, we replace the pure quark phase by an analytic expression (see discussion below). The pure confined hadronic phase exists below baryon number density $0.246 \mathrm{fm}^{-3}$ while pure deconfined quark matter exists above $0.862 \mathrm{fm}^{-3}$ $\left(1 \mathrm{fm}=10^{-13} \mathrm{~cm}\right)$. In between these two phases, a mixed quarkhadron phase exists (see Fig. 1, which displays the pressure $P$ vs. energy density $\epsilon$ relation for this EOS).

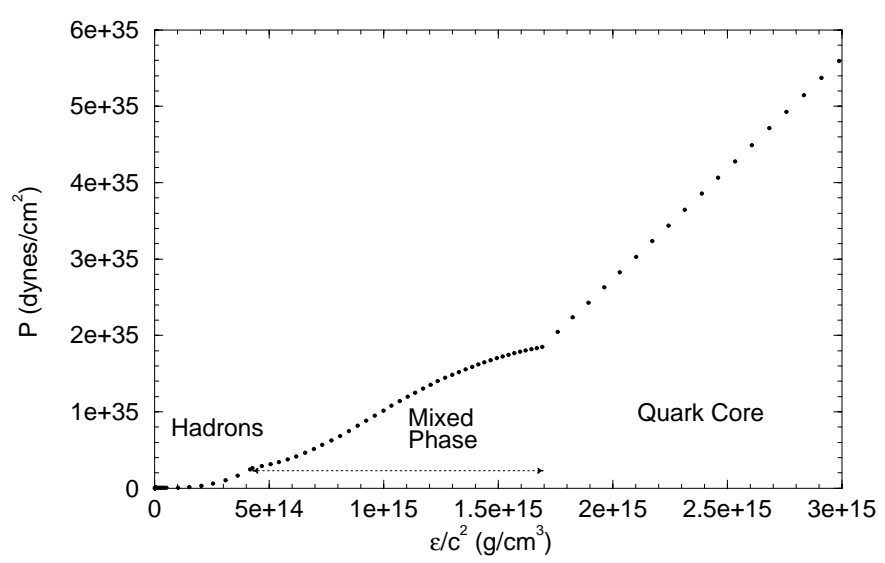

Fig. 1. Tabulated points of pressure $P$ vs. energy density $\epsilon$ for the quark deconfinement EOS.

When computing nonrotating models with the original tabulated EOS, using the 2-D code by Stergioulas \& Friedman (1995), we noticed that for central energy densities near the central energy density where the pure quark phase appears, the gravitational mass vs. central energy density plot shows an unusual behaviour (see Fig. 2). There is a local maximum followed by a local minimum and the mass increases again, before it reaches the maximum mass allowed by the generalrelativistic radial instability (see Shapiro \& Teukolsky 1983). Such a behaviour is not present, for exactly the same tabulated EOS, when using a simple 1-D code that integrates the well-known radial TOV equations of structure for non-rotating models. Thus, the oscillatory behaviour of the results obtained with the 2-D code for nonrotating models is a numerical effect. In the 2-D code, the angular behaviour of all variables is expanded as a sum of Legendre polynomials $P_{l}(\mu)$ of argument $\mu=\cos \theta$. At discontinuities, such as the surface of the star (where e.g. the velocity profile is discontinuous), the accuracy of the code is reduced due to the Gibbs phenomenon (see Nozawa et al. 1998). Similarly, when the pure quark phase appears at the center of the star, discontinuities appear in the numerical solution, as the EOS features an abrupt change of slope (see Fig. 1). In addition, as the EOS table only contains a limited number of points, the interpolation methods (such as 4-point Lagrange interpolation or Hermite polynomial interpolation) that are used in our code, do not avoid an oscillatory behaviour in the interpolated quantities, near the discontinuity in the slope of the EOS. It appears that, in the 2-D code, such an interpolation error enhances the Gibbs phenomenon at the center of the star, when the pure quark core appears, resulting in the oscillatory behaviour in Fig. 2.

To solve this numerical problem, we replace the part of the EOS that represents the pure quark phase by an analytic expression. In fact, we find that this part can be represented rather accurately by the following linear relation:

$$
\begin{aligned}
P\left[\text { dynes } / \mathrm{cm}^{2}\right]= & 1.85240 \times 10^{35} \\
& +2.885209 \times 10^{20}\left(\epsilon / c^{2}-1.69100 \times 10^{15}\right)
\end{aligned}
$$

where $P$ is pressure, $\epsilon$ is energy density and $c$ is the speed of light (in CGS units). In this way, the tabulated EOS is used only in the region below the pure quark phase and no interpolation is done through the phase transition, thus eliminating the problem of the oscillatory behaviour. For nonrotating models, this refined tabulated/analytic EOS now reproduces the same mass vs. central energy density relation with the 2-D code, as the original tabulated EOS with the 1-D code (see Fig. 2). Notice that for this comparison we avoid interpolating through the region where the pure quark appears, by choosing points either before or after the phase transition only. This increases the accuracy of computed models (as compared to interpolating through all points in the table) even with the 1-D code.

Thus, we have verified that replacing the pure quark region of the original tabulated EOS by the analytic expression (2) eliminates numerical problems in the 2-D code, while still representing the same EOS (with sufficiently high accuracy).

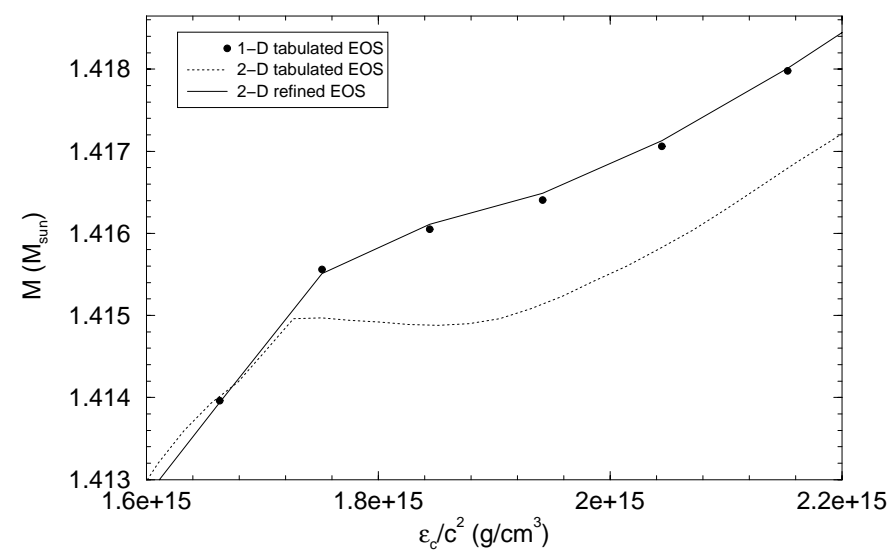

Fig. 2. Gravitational Mass $M$ vs. central energy density $\epsilon_{c}$ for nonrotating models, computed by various methods: using the original tabulated EOS in the 2-D code produces an oscillatory behaviour (dotted line). In contrast, using the refined tabulated/analytic EOS in the 2-D code (solid line) gives results in agreement with the original tabulated EOS in the 1-D code (when interpolation through the phase transition is avoided) (filled circles).

\section{Spin-down sequence}

We study several evolutionary sequences of pulsars, that differ from each other in the assumed constant baryonic mass. 
For a baryonic mass of $M_{0}=1.555 M_{\odot}$ we obtain a sequence that terminates at the maximum mass nonrotating model allowed by the EOS. This sequence is the limiting sequence between normal pulsars and supramassive pulsars and is very similar to the sequence studied in GPW. We assume that a compact star with a baryonic mass of $M_{0}$ is rapidly rotating. The compact star subsequently spins down due to e.g. magnetic braking and/or gravitational radiation emission, and the sequence ends either at the nonrotating limit (for normal pulsars), or the pulsar encounters the axisymmetric instability limit to black hole collapse (see e.g. Cook et al. 1984, for examples of such sequences for various realistic EOSs). The sequences are constructed using a highly accurate numerical code (rns, Stergioulas \& Friedman 1995) that computes exact numerical solutions of rapidly rotating stars in general relativity, without any approximation (apart from the truncation errors of the numerical scheme). Solutions are obtained on a 2-D numerical grid, with typical grid-size of $800 \times 400$ or $1200 \times$ 400 (radial $\times$ angular) points. Each model is defined by its central energy density and polar to equatorial axis ratio. Apart from the requirement of high accuracy for each individual model, essential to the construction of a constant-baryon number sequence, is the accuracy with which a model of a given baryonic mass is located. To this end, we construct various models by keeping the central energy density fixed, varying only the axes ratio and use a robust root-finding method to locate a model of given baryonic mass within a specified accuracy. The root-finding method of our choice is Ridders' method (Ridders 1979), since the procedure is guaranteed to stay within the initial brackets (avoiding unphysical parameters) and since it has a relatively high order of convergence $(\sqrt{2})$. In practice, about 10-12 individual models are required for the root-finding method to converge to a model of given baryonic mass, with a relative accuracy of $10^{-9}$ (the accuracy in computing the baryonic mass for an individual model is of the order of $10^{-5}$, but this does not limit the root-finding method to converge to a specified value with higher relative accuracy).

\section{Moment of inertia and gravitational mass}

We compute several physical parameters of the equilibrium models, the moment of inertia $I$, gravitational mass $M$, angular momentum $J$ and angular velocity $\Omega$ being the most relevant for our present study. We find that the behaviour of the moment of inertia depends on the baryonic mass of the sequence. Along a normal sequence with $M_{0}=1.551 M_{\odot}$, which is very close to the maximum mass nonrotating limit, but still below it, the change in the moment of inertia, as the star spins down, is shown in Fig. 3, which plots $I$ as a function of $\Omega$ (solid line). Using our refined tabulated/analytic EOS, described above, the behaviour of the moment of inertia is seen to be regular, i.e. $I$ is a single-valued function of $\Omega$. We notice that an apparent backbending appears with our 2-D code for this sequence, when the EOS in the original tabulated form (and interpolation through all points in the table) is used (dashed line in Fig. 3). This is an indication that the backbending of the moment of inertia

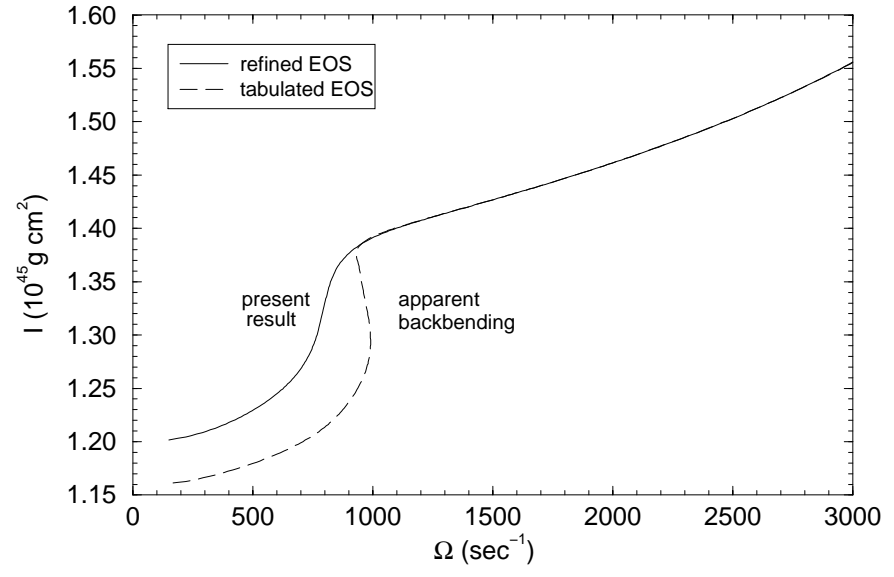

Fig. 3. Moment of Inertia $I$ vs. angular velocity $\Omega$, along the normal spin-down sequence with $M_{0}=1.551 M_{\odot}$. The original tabulated EOS produces an apparent backbending in the moment of inertia (dashed line) while the refined tabulated/analytic EOS yields the evolution of the moment of inertia without a backbending behaviour.

is sensitive to the available points in the EOS table and/or to truncation of rotational effects on the structure of the relativistic star.

Another important equilibrium property that we monitor along the equilibrium sequence is the gravitational mass. In Fig. 4, the gravitational mass $M$ is plotted as a function of central energy density $\epsilon_{\mathrm{c}}$. The solid line corresponds to the refined tabulated/analytic EOS and shows a monotonous decrease of the gravitational mass during spin-down. This normal behaviour corresponds to the monotonous behaviour of the moment of inertia in Fig. 3. In contrast, when one uses the original tabulated form of the EOS, the gravitational mass appears to oscillate as a function of angular velocity. This oscillatory behaviour is not correct, as it is the result of numerical truncation errors, due to the interpolation through the phase transition in the original tabulated EOS.

The oscillatory behaviour obtained with the original tabulated EOS deserves some further attention. As we will show, if it were the correct behaviour, it would correspond to a part of a sequence being unstable to quasi-radial perturbations: In this sequence, before the quark core appears, the equilibrium models are stable, while the star loses kinetic energy $T$ and angular momentum $J$, and the radius decreases only due to the decrease in rotational flattening of the star during spin down. When the pure quark core appears at the center of the star, the radius starts decreasing significantly, as the EOS becomes softer. Looking at models with larger central densities along the equilibrium sequence, the angular velocity $\Omega$, angular momentum $J$, kinetic energy $T$, absolute value of gravitational binding energy $|W|$ and gravitational mass $M$, all increase. Thus, a star cannot continue evolving along this uniformly rotating sequence to the expense of the star's available mass-energy. In Fig. 4 (dashed lined) a turning point appears and the gravitational mass increases with increasing central density. This is a typical case of the onset of the instability to the fundamental axisymmetric, quasi-radial mode of oscillation, in rotating relativistic stars, 


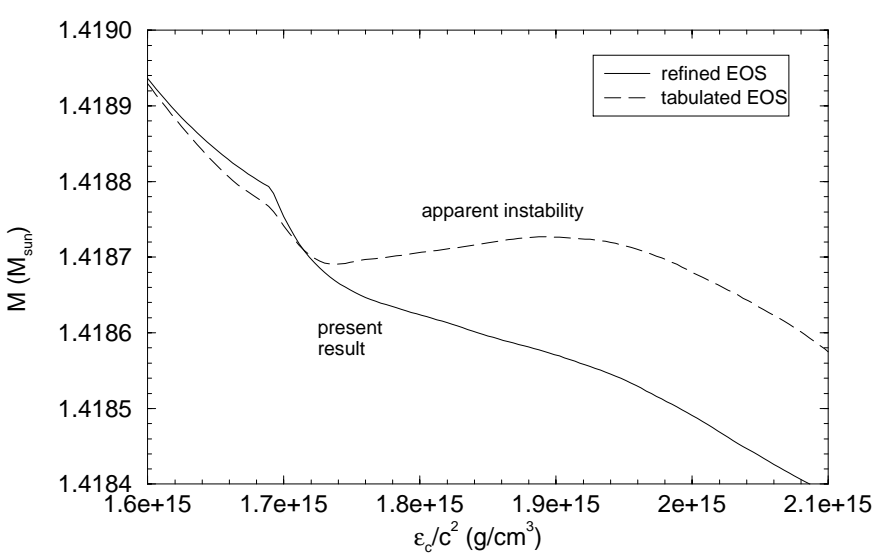

Fig. 4. Gravitational Mass $M$ vs. central energy density $\epsilon_{\mathrm{c}}$ along the normal spin-down sequence with $M_{0}=1.551 M_{\odot}$. Using the original tabulated EOS produces two turning points (that would correspond to an unstable region) (dashed line) while the refined tabulated/analytic EOS produces a monotonous behaviour.

as shown by Friedman et al. (1988) (hereafter FIS). The turning-point criterion for the onset of instability was first proved in FIS for a constant-angular-momentum sequence. Along such a sequence, an extremum in the $M$ vs. $\epsilon_{c}$ curve separates the regions of stable and unstable stars, i.e.

$\left(\frac{\partial M}{\epsilon_{\mathrm{c}}}\right)_{J}=0 \Leftrightarrow$ onset of instability,

for a sequence of uniformly rotating stars obeying a oneparameter $P=P(\epsilon)$ equation of state. Such a criterion was then proved by Cook et al. (1992) to also exist for sequences of constant baryonic mass $M_{0}$ :

$\left(\frac{\partial M}{\epsilon_{\mathrm{c}}}\right)_{M_{0}}=0 \Leftrightarrow$ onset of instability.

The instability to the fundamental quasi-radial mode of oscillation is first a secular one, i.e. it proceeds on a viscous timescale. It has been shown, however, that a dynamical instability sets in near the secular instability point (see Shibata et al. 2000). Thus, if the star would evolve along the dashed curve in Fig. 4, reaching the secular instability point, it would first evolve away from the uniformly-rotating equilibrium sequence, on a viscous timescale, driven by magnetic braking torques and/or gravitational radiation reaction and could then also become dynamically unstable, in which case it would suddenly contract. Eventually, the star would bounce at higher densities, when reaching again a stable branch in the equilibrium sequence (after the second turning point in Fig. 4).

We find that, when computing sequences of constant baryonic mass, the behaviour of the gravitational mass can be used as a guide to distinguish whether the sequence has been computed accurately: a behaviour such as the apparent instability described above, indicates a strong influence of numerical errors in the computation.

Looking at other constant baryonic mass sequences, with baryonic mass between $M_{0}=1.551 M_{\odot}$ and $M_{0}=1.555 M_{\odot}$

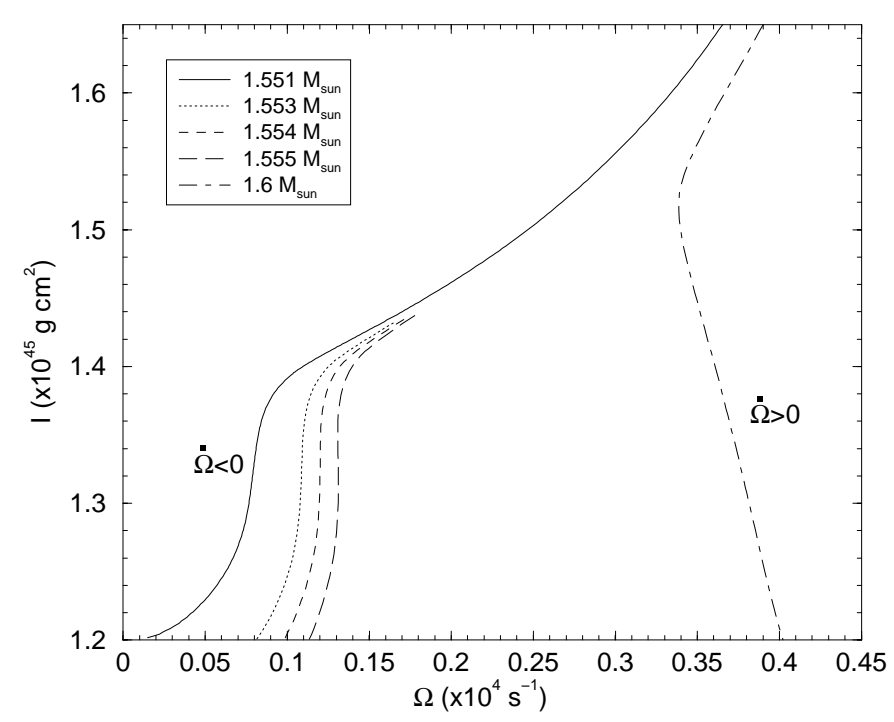

Fig. 5. Moment of Inertia $I$ vs. angular velocity $\Omega$, along several spindown sequences. Normal sequences $\left(M_{0}<1.555 M_{\odot}\right)$ do not show a backbending behaviour, while for supramassive sequences, such as the $M_{0}=1.6 M_{\odot}$ sequence, the backbending (spin-up) behaviour is dominant.

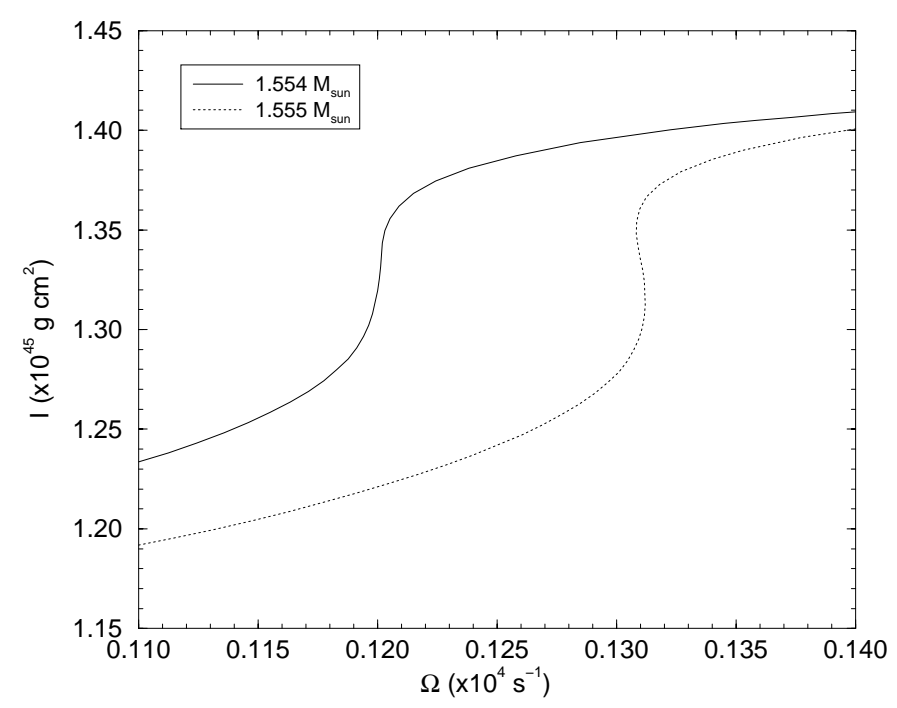

Fig. 6. Close-up of Fig. 5. A small backbending behaviour first occurs for the limiting sequence of $M_{0}=1.555 M_{\odot}$ that divides normal from supramassive stars and terminates at the maximum-mass nonrotating model allowed by the given EOS.

(see Fig. 5), we see that the moment of inertia gradually approaches a vertical behaviour $(\partial I / \partial \Omega \rightarrow \infty)$, as the limiting sequence that divides normal from supramassive stars is approached. A very small backbending (spin-up) behaviour is first observed for the limiting sequence of $M_{0}=1.555 M_{\odot}$ (and clearly not for e.g. $M_{0}=1.554 M_{\odot}$, see Fig. 6). For larger baryonic masses (supramassive sequences) the backbending behaviour becomes stronger and for e.g. a baryonic mass of $M_{0}=1.6 M_{\odot}$ it becomes the dominant behaviour, i.e. once the 
quark core appears, the star only spins-up with angular momentum loss, for the rest of its lifetime.

To summarize, for the particular EOS that we have studied in this paper, normal pulsars do not feature a spin-up era during phase transition, while supramassive pulsars only spin up after the phase transition. The limiting sequence that divides normal from supramassive pulsars shows a very small era of spin-up and appears to be the dividing line between stars that can spin up and stars that only spin down.

\section{Thermodynamic consistency}

In the previous sections we demonstrated that the refined tabulated/analytic EOS produces a constant baryonic mass sequence with equilibrium properties that are in sharp contrast to the equilibrium properties computed on the basis of the original tabulated EOS. In this section we will use an independent check to verify that the equilibrium properties obtained with the refined EOS are indeed the physically acceptable ones, while the equilibrium sequence obtained with the tabulated EOS suffers from numerical errors.

The independent check of the accuracy of the computed equilibrium properties is provided by the use of a relation that was first proved by Ostriker \& Gunn (1969) in Newtonian theory and then derived in general relativity by Bardeen (1970).

Along a sequence of uniformly rotating models of constant baryonic mass, changes in $M$ and $J$ are related by

$c^{2} \mathrm{~d} M=\Omega \mathrm{d} J$

which can be regarded as an expression of the first law of thermodynamics for such sequences.

We have evaluated the above relation numerically along the obtained sequences of equilibrium models (using sufficiently

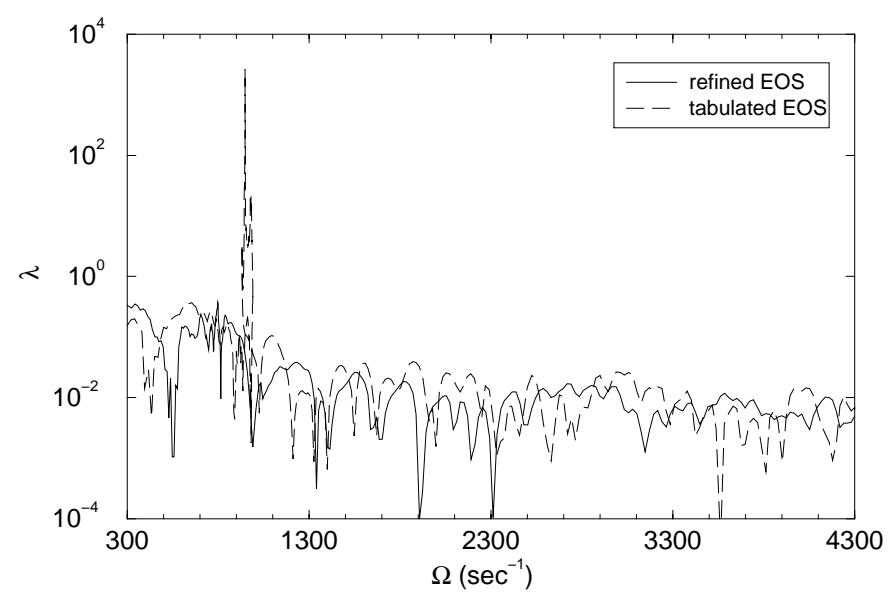

Fig. 7. Error indicator $\lambda$, constructed with the use of the thermodynamic consistency relation (see text) for the original and for the refined EOSs, along the sequence of rotating models undergoing the quark-hadron phase transition, with $M_{0}=1.551 M_{\odot}$. The refined EOS gives significantly more accurate models in the region where the pure quark core appears. many individual models to ensure an accurate evaluation of the numerical derivative). We define an error indicator, $\lambda$, as

$$
\lambda=\left|1-\frac{c^{2} \mathrm{~d} M}{\Omega \mathrm{d} J}\right| .
$$

The evaluation of $\lambda$ along the evolutionary sequence of $M_{0}=$ $1.551 M_{\odot}$ (Fig. 7), shows that the above relation is satisfied with an accuracy of $\sim 1 \%$ for models with central energy densities smaller than the energy density at which the pure quark-core appears. After the appearance of the pure quark core, numerical errors result in huge values of $\lambda$, when using the original tabulated EOS. With the use of the refined tabulated/analytic EOS, these errors are reduced significantly to an acceptable level and diminish as the resolution is increased.

The above check confirms that the refined EOS indeed produces physically acceptable results, while the original tabulated EOS produces results that are dominated by numerical error in the region in question.

\section{Behaviour of the observable braking index}

If a compact star is detected as a pulsar and one can measure the first two time-derivatives $\dot{\Omega}, \ddot{\Omega}$ of its angular velocity, then one can define an observational braking index

$n=n(\Omega) \equiv \frac{\Omega \ddot{\Omega}}{\dot{\Omega}^{2}}$.

In the Newtonian, slow-rotation limit, the spin-down of a pulsar is usually modeled in the form of a power law (see Shapiro \& Teukolsky 1983), namely assuming that only kinetic energy is lost and that the rate of loss is proportional to some power of the angular velocity of the star, i.e.

$\frac{\mathrm{d} T}{\mathrm{~d} t}=\kappa \Omega^{\alpha}$,

where $\kappa<0$ and $\alpha>0$ are usually assumed to be real constants. With these assumptions, the braking index is equal to $n=\alpha-1$. For example, it is assumed that, for magnetic braking, $\dot{T} \sim \Omega^{4}$, which yields an expected braking index $n=3$, while, for gravitational wave emission, $\dot{T} \sim \Omega^{6}$, which yields $n=5$. For slowly rotating pulsars, the above considerations are, in most cases, appropriate. However, for rapidly rotating pulsars one has to take into account the rotational flattening of the star. To a first approximation, this can be done considering rotational effects up to order $O\left(\Omega^{2}\right)$. Glendenning (1997) gives the rotationally corrected $\dot{\Omega}$ and braking index $n(\Omega)$ for a sequence of uniformly rotating stars, assuming the spin-down law of Eq. (7). As we will show here, the expressions given in Glendenning (1997) are incomplete, in the sense that the derivation is not fully consistent to $O\left(\Omega^{2}\right)$, but misses additional contributions of the same order.

The energy lost in the form of electromagnetic or gravitation radiation is not only to the expense of the star's kinetic energy (which would be the case only in the $O(\Omega)$ slow-rotation approximation) but to the expense of the star's total mass-energy (gravitational mass). In the Newtonian limit, 
this principle has been applied to the spin-down of rapidly rotating neutron stars by Finn \& Shapiro (1990) and by Spyrou \& Stergioulas (2001). In relativistic stars, the total mass-energy is

$M c^{2}=M_{0} c^{2}+U+T+W$

(see Friedman et al. 1986) where, additionally, $U$ is the internal energy. We thus adopt the following natural generalization of Eq. (7) in general relativity:

$c^{2} \frac{\mathrm{d} M}{\mathrm{~d} t}=\kappa \Omega^{\alpha}$.

The kinetic energy in relativity is defined as $T=(1 / 2) J \Omega$, so that

$\frac{\mathrm{d} T}{\mathrm{~d} t}=\frac{1}{2}\left(c^{2} \frac{\mathrm{d} M}{\mathrm{~d} t}+\frac{\mathrm{d} \Omega}{\mathrm{d} t} J\right)$

Expressing this in terms of the moment of inertia, which is defined as in Friedman et al. (1986), $I=J / \Omega$, and with the help of Eq. (4), we find

$\frac{\mathrm{d} T}{\mathrm{~d} t}=\left(c^{2} \frac{\mathrm{d} M}{\mathrm{~d} t}-\frac{1}{2} \Omega^{2} \frac{\mathrm{d} I}{\mathrm{~d} t} J\right)$

Equation (11) shows that, in a rapidly rotating star, under the above assumptions, the time derivatives of the kinetic energy and gravitational mass differ by an $O\left(\Omega^{2}\right)$ term that is proportional to the time-derivative of the moment of inertia. Notice that this expression is exactly valid for any rotation rate (even for stars rotating at the mass-shedding limit) and not only in an $O\left(\Omega^{2}\right)$ approximation. The relations derived in Glendenning (1997) for the braking index and time-derivative of the angular velocity in the $O\left(\Omega^{2}\right)$ approximation, are missing the contribution from the $O\left(\Omega^{2}\right)$ term on the r.h.s. of Eq. (11).

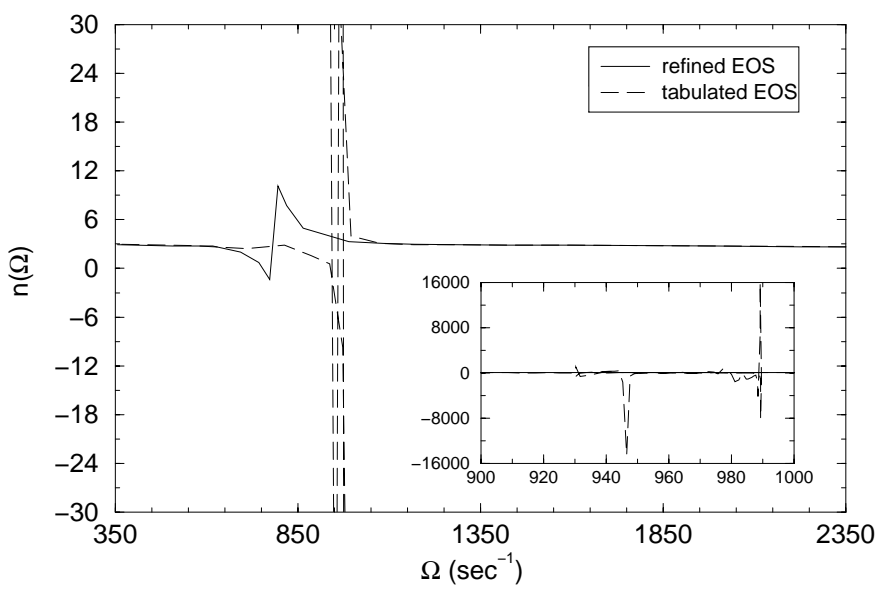

Fig. 8. Observational braking index $n(\Omega)$ along the spin-down sequence with $M_{0}=1.551 M_{\odot}$. The original tabulated EOS yields a singular behaviour at two nearby values of the angular velocity (see inset). The refined EOS does not yield a singular behaviour, but only a much weaker increase and corresponding decrease (with respect to the canonical value of $n=3$ ) at the pure quark core appearance.
With the above assumptions, it is easy to show that Eqs. (5.18) and (5.20) in Glendenning (1997) are replaced by

$\dot{\Omega}=\frac{\kappa}{I} \Omega^{\alpha-1}\left(1+\frac{\Omega I^{\prime}}{I}\right)^{-1}$,

where $I^{\prime}=\mathrm{d} I / \mathrm{d} \Omega$ and

$n(\Omega)=\alpha-1-\frac{2 I^{\prime} \Omega+I^{\prime \prime} \Omega^{2}}{I+I^{\prime} \Omega}$.

We have verified that Eq. (13) is the correct one, by numerically computing the braking index as obtained above and comparing it to the direct computation of the braking index, using the definition Eq. (6). The two numerical results agree well even close to the mass-shedding limit. In contrast, the expression for $n(\Omega)$ in Glendenning (1997) shows a large disagreement with the result obtained from the definition of $n(\Omega)$ for rapidly rotating stars. This difference between the two expressions is significant also in the case of supramassive sequences.

In Fig. 8, the observational braking index $n(\Omega)$ is shown as a function of angular velocity (computed along the normal spin-down sequence with $M 0=1.551 M_{\odot}$ ), assuming that the spin-down is only due to magnetic braking. The computed $n(\Omega)$ is roughly $n \simeq 3$ before the pure quark core appearance. During the phase transition, the braking index rises to roughly $n \simeq 10$ and then decreases to a value of roughly $n \simeq-2$, before returning to the canonical value of $n \simeq 3$. This behaviour is qualitatively and quantitatively different from the singular behaviour in the braking index obtained with our code when one uses the original tabulated EOS (see inset in Fig. 8).

\section{Initial period of PSR J0537-6910}

PSR J0537-6910 was discovered in SNR N157B by Marshall et al. (1998). It has a spin period of only $16 \mathrm{~ms}$, the fastest of all Crab-like pulsars, and a period derivative of $\dot{P}=5.13 \times$ $10^{-14} \mathrm{ss}^{-1}$. Both the characteristic age $\tau=P /(2 \dot{P})$ and the age estimate for SNR N157B suggest that the pulsar is 5000 years old. An upper limit on the age of the pulsar based on observations of $\mathrm{H} \alpha$-emitting gas in N157B (Chu et al. 1992), is 20000 years. Marshall et al. show that, if the braking index is $n=3$ and the pulsar age is 5000 years, then the pulsar must have been born with an extremely small (even sub-millisecond) period. Such small initial periods in pulsars associated with supernova remnants have not been detected to date. There are theoretical arguments, based e.g. on the possible occurrence of gravitational-wave induced instabilities in rapidly rotating relativistic stars (see e.g. Stergioulas 1998; Andersson \& Kokkotas 2001 for reviews) that suggest a pulsar initial period (shortly after the compact star is born) of at least several ms. How can this theoretical expectation be compatible with the observed properties of PSR J0537-6910? One possible solution would be that the actual braking index for this pulsar is closer to $n=2$ (for the upper limit on age of 20000 years, a very low braking index of $n \simeq 1$ is required).

Here we show that an initial period of several $\mathrm{ms}(6 \mathrm{~ms}$ in our example) can be obtained for a braking index of $n=3$, if the appearance of a pure quark core is considered. We use the 
same EOS and the $M_{0}=1.551 M_{\odot}$ constant baryonic mass sequence mentioned in previous sections and numerically integrate Eq. (12). We find that the age of the pulsar can be expressed in the following integral form

$t_{\mathrm{age}}=-\frac{1}{\kappa} \int_{\Omega_{i}}^{\Omega} \frac{I+\Omega \mathrm{d} I / \mathrm{d} \Omega}{\Omega^{\alpha-1}} \mathrm{~d} \Omega$,

where $\Omega_{i}$ is the initial angular velocity, or, equivalently,

$t_{\text {age }}=-\frac{1}{\kappa} \int_{J_{i}}^{J} \Omega^{1-\alpha} \mathrm{d} J$,

where $J_{i}$ is the initial angular momentum. For numerical integration, it is more convenient to introduce a dimensionless central energy density $\tilde{\epsilon}_{\mathrm{c}}=\left(\epsilon_{\mathrm{c}} / c^{2}\right) /\left(10^{15} \mathrm{~g} / \mathrm{cm}^{3}\right)$ as the integration parameter, and evaluate

$t_{\text {age }}=-\frac{1}{\kappa} \int_{\tilde{\epsilon}_{\mathrm{c}}(i)}^{\tilde{\epsilon}_{\mathrm{c}}} \Omega^{1-\alpha} \frac{\mathrm{d} J}{\mathrm{~d} \tilde{\epsilon}_{c}} \mathrm{~d} \tilde{\epsilon}_{\mathrm{c}}$.

The result of the numerical integration is shown in Fig. 9, which displays the age of the pulsar as a function of the central energy density. For a current spin of $16 \mathrm{~ms}$ and a pulsar age of 5000 years, the initial spin is obtained to be $6 \mathrm{~ms}$. Thus, even an $n=3$ braking index can yield an initial spin that is in agreement with theoretical expectations, provided the occurrence of a pure quark core is taken into account. In the above example, a particular choice of EOS and baryonic mass was made. Different choices would lead to different initial spin period estimates. However, the qualitative effect of the presence of a pure quark core is to increase (with respect to an EOS without a phase transition) the computed initial spin period.

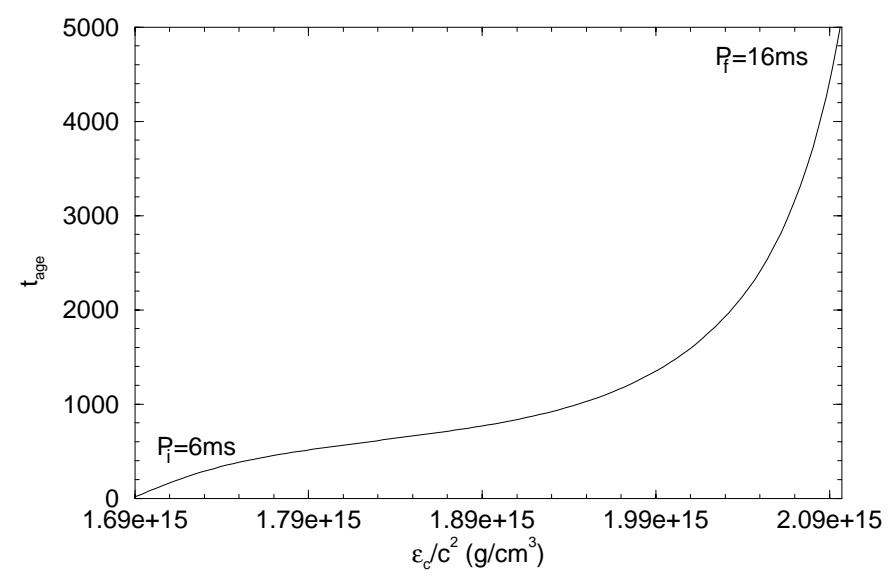

Fig. 9. Computed age of PSR J0537-6910 as a function of its central energy density. For a current age of 5000 years, the initial spin period is $6 \mathrm{~ms}$, significantly larger than in the absence of a phase transition.

\section{Event Rate}

An important parameter for the successful detection of a phasetransition signal in the braking index of spinning-down pulsars is the event rate, which is determined by several important factors. Let's assume that pulsars are born with an initial period of only a few milliseconds. Since normal pulsars have a magnetic field strength of $\sim 10^{12} \mathrm{G}$, they would quickly spin down to much larger periods due to magnetic dipole radiation. Thus, if the baryonic mass of a newly-born pulsar is such that the phase transition appears at a short rotational period, then the phase transition signal will not last for an extended period of time to have a realistic chance of being detected. Notice that no young, Crab-like pulsar with period shorter than $16 \mathrm{~ms}$ has been observed, which is indicative of the very small event rate that should be expected for such an observation.

On the other hand, if pulsars are born with rotational periods larger than, say, $6 \mathrm{~ms}$, then a phase transition can occur during their lifetime only if their baryonic mass falls within an extremely small range of values. To illustrate this point, we plot in Fig. 10 the constant baryonic mass sequence considered in Sect. 3 and the corresponding sequence of nonrotating models. The vertical dashed line specifies the central density at which the pure quark core appears. It crosses the constant-baryonicmass sequence at a model with rotational period of $6 \mathrm{~ms}$ and gravitational mass of $1.419 M_{\odot}$. Notice that the gravitational mass of a nonrotating star with central energy density equal to that of the quark core appearance $\left(1.691 \times 10^{15} \mathrm{~g} / \mathrm{cm}^{3}\right)$ is $1.414 M_{\odot}$. Thus, a pure quark core can appear in a spinning down pulsar with rotational period less than $6 \mathrm{~ms}$ only if its mass falls within $\Delta M \sim 0.0025 M_{\odot}$ of the value of $1.4165 M_{\odot}$ (the sequence considered in this example). The probability for this to happen is obviously negligible.

The above arguments show that the only observed population remaining, in which one could hope for a significant event rate for the considered signal, are old millisecond pulsars, spun-up by accretion, with low magnetic field and spindown rate. The actual event rate will then depend on the massdistribution for this population and on the precise value of the central energy density at which the quark core appears. None of these parameters are currently known with sufficient accuracy to compute a reliable value for the event rate. In fact, the observational determination of the braking index is itself a difficult task and no braking index has been measured for any of the known millisecond pulsars, to date. However, should a millisecond pulsar become supramassive, due to mass-accretion, then there is a realistic chance for detecting the presence of a quark core in observations of the first time-derivative, $\dot{P}$, of the rotational period. Once the quark core appears and if the pulsar is sufficiently massive, then $\dot{P}<0$ for the rest of the pulsar's lifetime.

\section{Discussion}

Observational constraints for the properties of compact stars are currently still too weak to determine the correct equation of state of high-density matter. The presence of a quark/hadron deconfinement phase transition has been suggested by Glendenning et al. (1997) (GPW) to be observable in the braking index of pulsars. In this method, the observational signal comes from a spin-up era, originating from a backbending behaviour of the moment of inertia during the pure quark core appearance. 


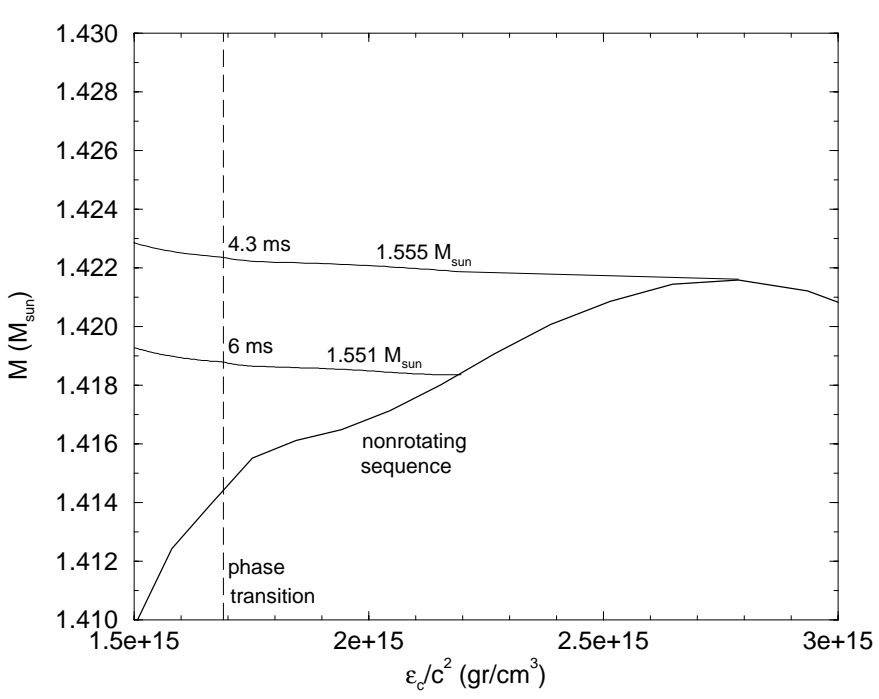

Fig. 10. Gravitational mass vs. central energy density $\epsilon_{\mathrm{c}}$ for the nonrotating sequence and for the spin-down sequence of constant baryonic mass $M_{0}=1.551 M_{\odot}$. The dashed vertical line indicates the central energy density at which a pure quark core appears. Slowly rotating normal pulsars can go through the phase transition, only if the gravitational mass falls within an extremely narrow range of values.

We have investigated the deconfinement phase-transition of spinning-down pulsars, using fully relativistic, rapidly rotating models. We find that, for normal pulsars, the star does not go through a spin-up era and the pure quark core appears without a backbending behaviour in the moment of inertia. The backbending behaviour first occurs for the limiting sequence of constant baryon mass that terminates at the maximum mass nonrotating model allowed by the EOS. In contrast, for sequences with larger mass (supramassive sequences) the backbending behaviour becomes dominant and pulsars only spin up, after a quark core appears.

From our numerical results, it is evident that the moment of inertia along a spin-down sequence is very sensitive to the appearance of phase transitions and must be computed with great care. In particular, we emphasize that low resolution and the restriction to a limited number of points in the EOS table (with interpolation through all points) can easily spoil the correct computation of the behaviour of the moment of inertia, at least in the present 2-dimensional code used in this paper.

The expected event rate of a phase-transition signal in the pulsar braking index is negligibly small for the population of normal pulsars. For the population of old millisecond pulsars, a reliable estimate for the event rate cannot be obtained, as it involves several uncertain factors and, in addition, the observational determination of braking indices in millisecond pulsars has not become technically possible, yet. However, if millisecond pulsars become supramassive, then the presence of a quark core could be detected by observing a negative first time-derivative of the rotational period. On the other hand, the absence of any such observation in the current population of known millisecond pulsars indicates that either large phase transitions do not occur, or that the EOS is sufficiently stiff to prevent accreting relativistic stars from becoming supramassive. A detailed study of phase transitions in supramassive models of relativistic stars, constructed with various EOSs, could provide interesting constraints on the equation of state at very high densities.

In order to describe the spin down of a pulsar due to magnetic dipole or gravitational radiation, we adopt a generalrelativistic version of the usual power-law assumed in the slowrotation limit. The energy loss is assumed to be to the expense of the total mass-energy of the star and not simply to the expense of the star's kinetic energy. In this way, we derive new expressions for the evolution of the angular velocity and braking index during spin down. As an application, assuming magnetic dipole braking only, the initial spin period of PSR J0537-6910 is obtained to be $6 \mathrm{~ms}$ (for the EOS and spin-down sequence considered in this paper). The presence of the deconfinement phase transition can thus give a more reasonable initial period for this pulsar, compared to previously obtained estimates.

PSR J0537-6910 exhibits the highest rate of large glitch events (6 in 3 years) (see Gotthelf et al. 2001) of all known Crab-like pulsars. At the same time, it is the most rapidly rotating Crab-like pulsar. If a pure quark core appears at small spin periods, then the quark-core fraction in such pulsars continuously changes during spin down, most probably contributing to the occurrence of large glitches. The fact that PSR J05376910 exhibits the highest glitch rate could be related to the fact that the phase transition may have happened more recently in its lifetime (according to the spin-down sequence used here) and its spin-down behaviour is still influenced by the gradual change of the quark core fraction during spin down. This suggestion has to be examined in more detail, as it could be a generic feature of phase transitions in the high-density EOS.

Acknowledgements. We thank Nils Andersson, Pawel Haensel and John L. Friedman for useful discussions and Emanuele Berti for a careful reading of the manuscript. We are grateful to Leszek Zdunik and Pawel Haensel for comparing numerical results of several evolutionary sequences, before publication. Finally, we are indebted to the referee, Prof. N. Glendenning, for critical comments that substantially improved the final version of this paper. This work has been supported by the EU Programme "Improving the Human Research Potential and the Socio-Economic Knowledge Base" (Research Training Network Contract HPRN-CT-2000-00137), KBN-5P03D01721 and the Greek GSRT Grant EPAN-M.43/2013555.

\section{References}

Andersson, N., \& Kokkotas, K. D. 2001, Int. J. Mod. Phys. D, 10, 381 Bardeen, J. M. 1970, ApJ, 162, 71

Chubarian, E., Grigorian, H., Poghosyan, G., \& Blaschke, D. 2000, A\&A, 357, 968

Christiansen, M., Glendenning, N. K., \& Schaffner-Bielich, J. 2000, Phys. Rev. C, 62, 025804

Cheng, K. S., Yuan, Y. F., \& Zhang, J. L. 2002, ApJ, 564, 909

Chubarian, E., Grigorian, H., Poghosyan, G., \& Blaschke, D. 2000, A\&A, 357, 968

Cook, G. B., Shapiro, S. L., \& Teukolsky, S. A. 1992, ApJ, 398, 203

Cook, G. B., Shapiro, S. L., \& Teukolsky, S. A. 1994, ApJ, 424, 823

Finn, L. S., \& Shapiro, S. L. 1990, ApJ, 359, 444

Friedman, J. L., Ipser, J. R., \& Parker, L. 1986, ApJ, 304, 115; Erratum 1990, ApJ, 351, 705 
Friedman, J. L., Ipser, J. R., \& Sorkin, R. D. 1988, ApJ, 325, 722

Glendenning, N. K. 1992, Phys. Rev. D, 46, 1274

Glendenning, N. K. 2002, personal communication

Glendenning, N. K. 1997, Compact Stars, Nuclear Physics, Particle Physics, and General Relativity (Springer-Verlag, New York)

Glendenning, N. K., Pei, S., \& Weber, F. 1997, Phys. Rev. Lett., 79, 1603

Gotthelf, E. V., Zhang, W., Marshall, F. E., Middleditch, J., \& Wang, Q. D. 2001, ASP Conf. Ser., preprint [astro-ph/0112021]

Heiselberg, H. 2001, Proc. of Compact Stars in the QCD Phase Diagram [astro-ph/0201465]

Heiselberg, H., \& Hjorth-Hensen, M. 1998, Phys. Rev. Lett., 80, 5485

Heiselberg, H., \& Hjorth-Hensen, M. 1999, ApJ, 525, L45

Marshall, F. E., Gotthelf, E. V., Zhang, W., Middleditch, J., \& Wang, Q. D. 1998, ApJ, 499, L179
Nozawa, T., Stergioulas, N., Gourgoulhon, E., \& Eriguchi, Y. 1998, A\&AS, 132, 431

Ostriker, J. P., \& Gunn, J. E. 1969, ApJ, 157, 1395

Pandharipande, V. R., \& Smith, R. A. 1975, Nucl. Phys. A, 237, 507

Ridders, C. J. F. 1979, IEEE Transactions on Circuits and Systems, vol. CAS-26, 979

Shapiro, S. L., \& Teukolsky, S. A. 1983, Black Holes, White Dwarfs and Neutron Stars (John Wiley \& Sons, New York)

Spyrou, N. K., \& Stergioulas, N. 2001, A\&A, 366, 598

Stergioulas, N. 1998, Living Rev. Relativity, 1, 8, http://www. livingreviews .org/Articles/Volume1/1998-8stergio/

Stergioulas, N., \& Friedman, J. L. 1995, ApJ, 444, 306

Shibata, M., Baumgarte, T., \& Shapiro, S. L. 2000, Phys. Rev. D, 61, 044012

Weber, F. 2001, J. Phys. G, 27, 465 